\title{
GLAM: An adaptive graph learning method for automated molecular interactions and properties predictions
}

\section{Yuquan Li}

Lanzhou University https://orcid.org/0000-0003-2756-0449

\section{Chang-Yu Hsieh}

Tencent

Ruiqiang Lu

Lanzhou University

\section{Xiaoqing Gong}

Lanzhou University

\section{Xiaorui Wang}

Lanzhou University

\section{Pengyong li}

Tsinghua University https://orcid.org/0000-0001-5971-046X

\section{Shuo Liu}

$$
\text { Lanzhou University }
$$

\section{Yanan Tian}

\section{Lanzhou University}

\section{Dejun Jiang}

tingjunhou@zju.edu.cn https://orcid.org/0000-0002-2035-5074

Jiaxian Yan

University of Science and Technology of China

\section{Qifeng Bai}

Lanzhou University https://orcid.org/0000-0001-7296-6187

Huanxiang Liu

Lanzhou University

\section{Shengyu Zhang}

Tencent

Xiaojun Yao ( $\nabla$ xjyao@lzu.edu.cn )

Lanzhou University 


\section{Keywords:}

Posted Date: January 6th, 2022

DOI: https://doi.org/10.21203/rs.3.rs-1172418/v1

License: (c) (1) This work is licensed under a Creative Commons Attribution 4.0 International License. Read Full License

Version of Record: A version of this preprint was published at Nature Machine Intelligence on June 23rd, 2022. See the published version at https://doi.org/10.1038/s42256-022-00501-8. 
1 GLAM: An adaptive graph learning method for automated

2 molecular interactions and properties predictions

4 Yuquan Li ${ }^{1,2, \dagger}$, Chang-Yu Hsieh ${ }^{2, \dagger}$, Ruiqiang Lu ${ }^{1}$, Xiaoqing Gong ${ }^{1}$, Xiaorui

$5 \quad$ Wang ${ }^{3}$, Pengyong Li ${ }^{4}$, Shuo Liu ${ }^{5}$, Yanan Tian ${ }^{5}$, Dejun Jiang ${ }^{6}$, Jiaxian Yan ${ }^{7}$,

6 Qifeng Bai ${ }^{8}$, Huanxiang Liu ${ }^{5}$, Shengyu Zhang ${ }^{2}, \&$ Xiaojun Yao ${ }^{1, *}$

$8 \quad{ }^{1}$ College of chemistry and chemical engineering, Lanzhou University, Lanzhou, China.

$9 \quad{ }^{2}$ Tencent Quantum Laboratory, Tencent, Shenzhen, China.

$10{ }^{3}$ State Key Laboratory of Quality Research in Chinese Medicines, Macau University of Science and

11 Technology, Macau, China.

$12{ }^{4}$ School of computer science and technology, Xidian University, Xian, China.

$13{ }^{5}$ School of pharmacy, Lanzhou University, Lanzhou, China.

$14{ }^{6}$ College of Computer Science and Technology, Zhejiang University, Hangzhou, China.

$15{ }^{7}$ School of Data Science, University of Science and Technology of China, Hefei, China.

$16{ }^{8}$ School of Basic Medical Sciences, Lanzhou University, Lanzhou, China.

17 These authors contributed equally to this work.

$18{ }^{*}$ Corresponding authors. E-mail: xjyao@1zu.edu.cn

19 
Abstract

Improving drug discovery efficiency is a core and long-standing challenge in drug

3 discovery. For this purpose, many graph learning methods have been developed to search

4 potential drug candidates with fast speed and low cost. In fact, the pursuit of high prediction

5 performance on a limited number of datasets has crystallized them, making them lose

6 advantage in repurposing to new data generated in drug discovery. Here we propose a

7 flexible method that can adapt to any dataset and make accurate predictions. The proposed

8 method employs an adaptive pipeline to learn from a dataset and output a predictor. Without

9 any manual intervention, the method achieves far better prediction performance on all tested

10 datasets than traditional methods, which are based on hand-designed neural architectures

11 and other fixed items. In addition, we found that the proposed method is more robust than

12 traditional methods and can provide meaningful interpretability. Given the above, the

13 proposed method can serve as a reliable method to predict molecular interactions and

14 properties with high adaptability, performance, robustness and interpretability. This work

15 would take a solid step forward to the purpose of aiding researchers to design better drugs

16 with high efficiency. 


\section{Introduction}

Drug discovery is a lengthy, costly, and complex process that plays a crucial role in human

3 health and well-being ${ }^{1,2}$. At present, experimental assays ${ }^{3}$ remain the most reliable approach to

4 screen compounds but cost too much. Although many computational methods ${ }^{4}$ are proposed to

5 estimate molecular interactions and properties and improve drug discovery efficiency, it is still a

$6 \quad$ tricky process.

7 Graph learning methods have the potential to improve drug discovery efficiency dramatically

8 for their ability to amplify insights available from existing drug-related datasets ${ }^{5}$. Using the insights

9 to predict molecular interactions and properties ${ }^{6,7}$ is a key to finding potential drug candidates from

10 the vast chemical space with extremely fast speed and low cost. On the other hand, molecular

11 generation ${ }^{8,9}$ based on the insights can more efficiently traverse the vast chemical space to find

12 potential drug candidates. Accordingly, graph learning is becoming a rising area of interest within

13 the field of drug discovery ${ }^{10}$.

14 However, the pursuit of high prediction performance on a limited number of existing datasets

15 has crystallized them ${ }^{11-13}$, making them lose advantage in repurposing to new data generated in

16 drug discovery. In practice, a graph learning method tends to be made with a fixed architecture and

17 a fixed hyperparameter set to get the best performance on a dataset ${ }^{14}$. This heavily limits them in

18 repurposing to new data generated in drug discovery, which tend to be increasingly complex ${ }^{15}$.

19 Besides, most graph learning methods rely heavily on expert knowledge of deep learning to achieve

20 their claimed state-of-the-art results, or even cannot be reproduced ${ }^{16}$. Therefore, they are not

21 flexible and reliable enough.

More recently, a few works have been reported to address these problems above. MolMapNet

23 introduced an out-of-the-box deep learning method based on broadly learning knowledge-based 
1 representations to get reliable prediction performance on more datasets ${ }^{7}$. A recent work introduced

2 a neural architecture search based method to design neural architecture for molecular property 3 predictions ${ }^{11}$.

In this work, we proposed Graph Learning based Adaptive Machine (GLAM), a flexible

5 method that can adapt to any dataset and make accurate predictions without human intervention.

6 We fairly compared our proposed method with previously reported methods in terms of

7 adaptability and prediction performance. The results show that our proposed method can adapt to

8 all tested datasets exceptionally well and obtain far better prediction performance than reported

9 methods. On the other hand, we also investigated the robustness and interpretability of our

10 proposed method. We found that the proposed method is more roubst than tested methods and can

11 provide meaningful intepretability, making the proposed method a more reliable method.

\section{Results}

\section{Method Overview}

15 Our method utilizes an automated pipeline to learn from datasets and build a predictor, as

16 shown in Figure 1. Our method is mainly different from previous graph learning methods ${ }^{17-22}$,

17 which rely heavily on human experts to design architecture, tune model hyperparameters, select

18 the optimizer, and select the loss function. We creatively combine these four items into a

19 configuration and put potential configurations into a configuration space. Starting from this

20 configuration space, we perform configuration sampling, low-fidelity training based on sampled

21 configurations, configuration selection, high-fidelity training based on selected configurations,

22 predictor selection, and predictor blending to build a predictor. 
Building a Predictor by GLAM Automatically

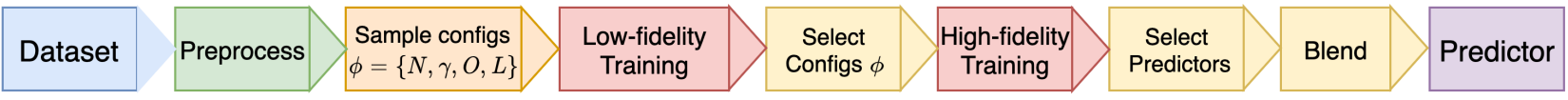

human decision free

Building a Predictor by Human Expert

bad performance

\begin{tabular}{|c|c|c|c|c|c|c|c|c|}
\hline Dataset & Preprocess & $\begin{array}{c}\text { Design } \\
\text { Architecture } N\end{array}$ & $\begin{array}{c}\text { Set } \\
\text { Hyperparams } \gamma\end{array}$ & $\begin{array}{c}\text { Select } \\
\text { Optimizer } O\end{array}$ & $\begin{array}{c}\text { Select } \\
\text { Criterion } L\end{array}$ & $\begin{array}{l}\text { Train } \\
\text { Model }\end{array}$ & Evaluate & Predictor \\
\hline
\end{tabular}

human decision based

Figure 1. Overview of GLAM and traditional method.
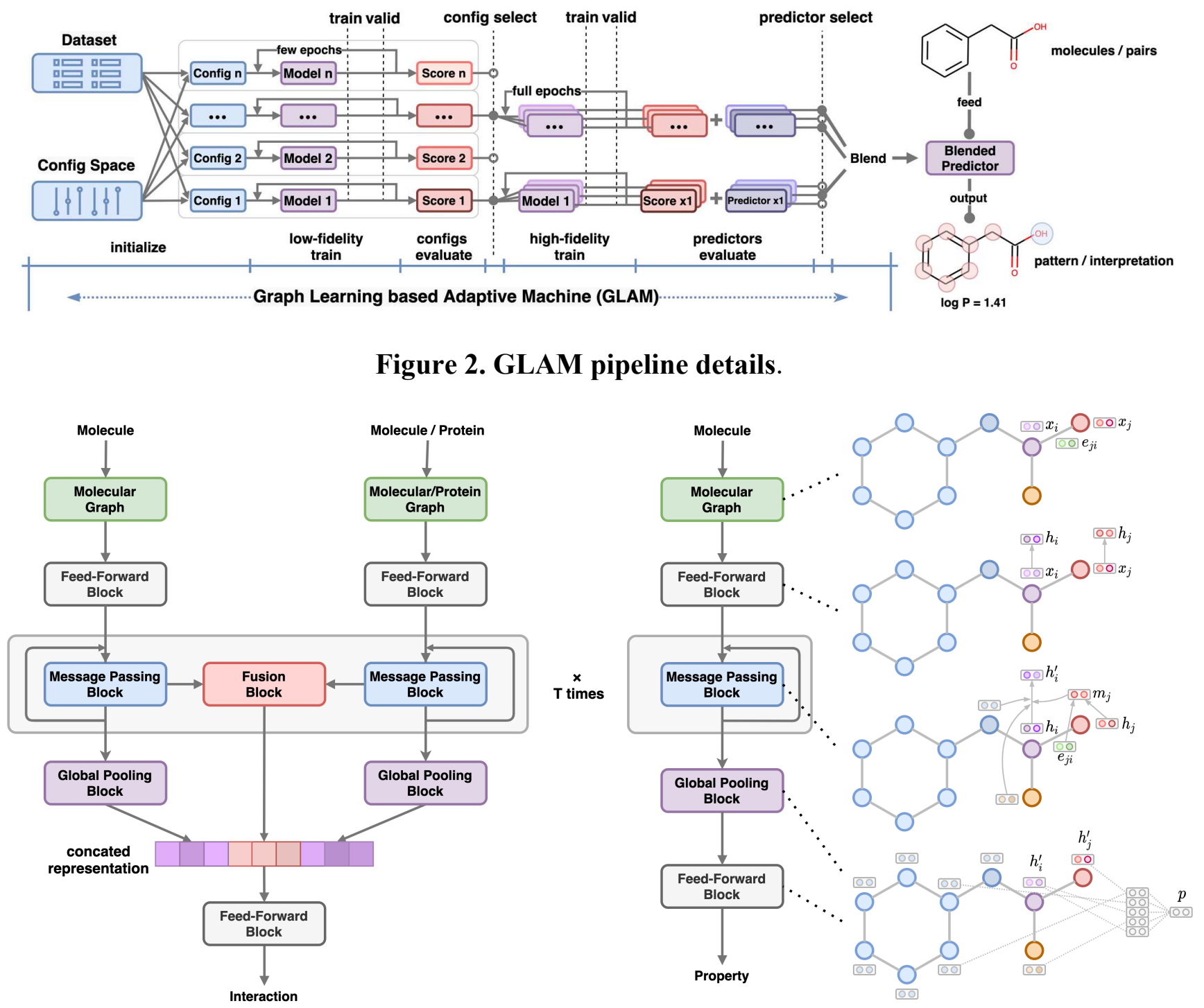

6 Figure 3. the general architectures for molecular interactions and properties prediction in GLAM. 
In detail, the method has a well-designed pipeline that is to achieve high adaptability,

2 prediction performance, and acceptable speed, as shown in Figure 2. Initially, GLAM samples a

3 lot of configurations from the configuration space. Then the dataset is fed to these configurations

4 for low-fidelity training, which produces evaluation scores to select the high-performance

5 configurations. In the next high-fidelity training, each previously selected configuration is fed with

6 the dataset to perform full epochs training with three different random seeds. Accordingly, the

7 evaluation scores of the high-fidelity training are used to select high-performance predictors.

8 Finally, all selected predictors are blended into a predictor.

9 We have designed two general architectures, one for molecular interaction and another for

10 molecular property, as shown in Figure 3. In practice, the input data are mapped to output by five

11 steps: (1) preprocess a molecule to a molecular graph, (2) linear transformation by feed-forward

12 block, (3) iterated convolution by message-passing block for T time-steps, (4) node reduction by

13 global pooling block, (5) linear transformation by final feed-forward block. Each block in the

14 general architecture is created with its own design space, as shown in Extended Data Figure 1.

15 Adapt to any dataset for high performance

16 GLAM was created to adapt to any dataset to obtain high prediction performance. To

17 investigate the adaptability and performance of our method, we compared its performance on 14

18 datasets with a range of representative traditional methods ${ }^{7,17-21,23-25}$. The types of tested datasets

19 include drug-protein interactions, drug-drug interactions, physical-chemistry property, bioactivity,

20 pharmacokinetics, and toxicity. Considering that different splits of datasets cause different

21 performances, we let all methods share the same splits of datasets for a fair evaluation. We also try

22 our best to adjust the parameters of all methods manually to achieve the best performance. Finally,

23 we run benchmarks on molecular interaction and molecular property datasets. 
Compared to all traditional methods, our proposed methods can adapt to datasets well and achieve promising prediction performance, as shown in Table 1, Table 2 and Extended Data

3 Table 1. (All tables can be found at the end of this manuscript.) Given the above, GLAM sets a new state-of-the-art on both molecular interactions and properties prediction. Relative to the best scores of reported results, the proposed method got an average $18.7 \%$ decrease of prediction error on 14 datasets than the best of traditional methods. In addition, GLAM can consistently achieve the best scores without human intervention. Therefore, GLAM is poised to be a flexible, reliable, and trustworthy method that works well across a wide range of activities in drug design.

\section{High robustness against molecular structure perturbation}

The following essential issue is about the robustness ${ }^{26}$ of the proposed method. Graph

11 learning methods usually are susceptible to outliers, and it can be disastrous if data is contaminated

12 with outliers. In fact, some safe-sensitive domains (such as healthcare) require more robustness of

13 a method as even a slight perturbation will lead to a wrong result with serious consequence 35.

14 Admittedly, the robustness of a graph learning method is also an essential issue.

15 To evaluate the robustness of our proposed method, we first introduce an algorithm named 16 property-slightly-affected structure perturbation (PASP). The PASP works by iteratively mutating

17 the molecular structure with small perturbations that do not significantly affect the properties of 18 the molecules.

Then we perform the PASP algorithm on a dataset named PhysProp ${ }^{28}$, consisting of 14,176

20 molecules structures with their corresponding properties $\log P$. To estimate the potential change of

$21 \log P$ upon mutations, we use $\mathrm{RDKit}^{29}$ to calculate the $\log P$ values before and after PASP as an

22 observer. Moreover, we limit an acceptable change (value change $<0.2$ before and after the PASP),

23 which can ensure that the PASP does not significantly affect the $\log P$ of molecules. In this 
experiment, The molecular structures whose similarity (before and after the mutation) of

2 fingerprint in the scale of $0.8-1.0,0.5-0.8$, and $0.3-0.5$ are marked as levels 1,2 , and 3 , respectively.

Table 3 shows that GLAM is less affected by the PASP and manifests higher robustness than conventional methods. We first run GLAM on the unperturbed train and validation sets to get a predictor. Then, we feed the test set to the predictor and migrate the testing to the three perturbed test sets. Compared with conventional methods, including $\mathrm{GCN}^{18}, \mathrm{GAT}^{19}$, $\mathrm{GIN}^{20,22}$, MPNN, the results show that all these methods suffer a significant performance drop, as shown in Table $\mathbf{3}$. In contrast, our method is less affected by PASP than conventional methods on all three levels of PASP. The robustness of the GLAM is most likely due to the model blending at the end of the

10 pipeline. The main idea of blending is to train several models and draw a final prediction from

11 averaging. So, perturbing the molecular structure may cause a molecule misclassified by a predictor

12 but not by all predictors.

\section{Interpretation cases}

To better understand the models in the predictor, we investigate its decision-making process

15 and interpret its learned knowledge. In the past, most machine learning models have long been 16 considered as black boxes. Previous works adopt attention mechanisms ${ }^{21,30,31}$ to aid interpretation

17 of the model. Here we explain the model from the hidden states by averaging and visualizing it, 18 which can directly utilize the information provided by the models in the predictor.

Extended Data Figure 2a shows some case studies of solubility prediction, which are

20 consistent with the intuition of chemists. Generally, hydroxyl and amino groups are considered to

21 be more hydrophilic and alkyl and halogen groups are considered to be more lipophilic. We select

22 and visualize some representative molecules from the PhysProp dataset. The atoms in the 23 hydrophilic group tend to be bluer in our visualization, which means their weights are closer to 1. 
1 In contrast, the atoms in the lipophilic group tend to be redder in our visualization, which means

2 their weights are closer to -1 . These observations are consistent with the intuition of chemists,

3 indicating that the models in predictor can detect essential atomic groups with clear interpretability

4 of results of solubility.

In the same way, we visualize some cases of drug-drug interaction identification as shown in

6 Extended Data Figure $2 \mathbf{b}$. We consider the interactions between sidenafil/udenafil and

7 nitrates(nicorandil/ isosorbide dinitrate) as cases. They are combined into four pairs of drug-drug

8 interactions and fed into models in predictor to visualize the decision process. (Typically, the

9 sildenafil/udenafil can selectively inhibit the phosphodiesterase type 5 (PDE5) targets in the human

10 body. The $\mathrm{N}$-methyl groups in the pyrazolorpyrimidone rings of sildenafil/udenafil are important

11 for the activity and selectivity of the PDE5. For the reasons above, combining sildenafil/udenafil

12 with nitrate drugs may lead to serious blood pressure reduction and heart diseases.) The

13 visualization results show the models in predictor pay more attention to the nitrates of isosorbide

14 dinitrate and nicorandil, and pay more attention to the N-methyl of sildenafil and udenafil. Thus,

15 Our visualization results are consistent with previous findings of drug interaction, indicating that 16 models in the predictor can provide deep insights into molecular interactions.

183 Discussion

We have shown that GLAM can adapt to all tested datasets well and make accurate

20 predictions automatically. In the past, the adaptability for new data has largely been ignored as

21 most researchers pay almost all attention to getting high prediction performance. Our well-designed

22 method can serve as a reliable method to predict molecular interactions and properties with high

23 adaptability, prediction performance, robustness and interpretability. Furthermore, the automated 
1 pipeline of our proposed method enables more researchers, even those who lack machine learning

2 experience, to make full use of the power of machine learning. These advantages of our proposed

3 method will greatly increase the acceptance of machine learning aided drug discovery.

54 Limitations and frontiers

Adaptive feature input can help the models in our proposed method to extract important and

7 sufficient representations. In this work, we only provide the graph model with the basic node

8 features, such as atomic/residual number. Adaptive feature input can be of great help to some

9 particular prediction jobs. Adding the feature decisions to configuration space might improve our

10 proposed method. An strategy that provides neither too little information nor too redundant

11 information would contribute a lot to the representation extraction process.

\section{Outlook}

14 Our proposed method is expected to advance and evolve the automated drug design ${ }^{1,32}$.

15 Recent advances such as chemical retrosynthesis predictions ${ }^{33,34}$ and molecular generations ${ }^{35-37}$

16 have laid the foundation for an automated drug design pipeline in the future. Automated drug

17 design or semi-automatic drug design will become a trend. The proposed method can serve as a

18 predictor generator contributing to the automated drug design. For further applications, our

19 proposed method can be repurposed to more scientific discovery fields, such as agrochemicals and

20 materials design. 


\section{$1 \quad$ Methods}

\section{Details of datasets}

We use LIT-PCBA ${ }^{38}$, BindingDB ${ }^{39}$, and DrugBank ${ }^{40}$ to evaluate our proposed method for

4 molecular interaction prediction. In LIT-PCBA, we selected four datasets of representative protein

5 based on the number of positive and negative samples. On the other hand, we used datasets in

6 MoleculeNet ${ }^{41}$ to evaluate the proposed method for molecular property prediction. MoleculeNet ${ }^{41}$

7 is a set of benchmarking datasets for molecular machine learning to achieve a fair performance

8 comparison.

9 LIT-PCBA is a virtual screening dataset consisting of fourteen targets, 7844 confirmed

10 active, and 407,381 confirmed inactive compounds ${ }^{38}$. BindingDB dataset contains 39,747 positive

11 examples and 31,218 negative examples from a public database ${ }^{39}$. DrugBank includes 1,850

12 approved drugs with 221,523 DDI positive labels ${ }^{40}$. The blood-brain barrier penetration (BBBP)

13 contains 2053 molecules on their permeability properties ${ }^{41}$. SIDER is a database of marketed drugs

14 and adverse drug reactions, which grouped into system organ classes for 1427 approved drugs ${ }^{41}$.

15 The BACE is a database consisting of binding results for a set of inhibitors of human $\beta$-secretase

161 with 1522 compounds $^{41}$. Tox 21 contains qualitative toxicity measurements for 8014 compounds

17 on 12 different targets, including stress response pathways and nuclear receptors ${ }^{41}$. ToxCast is

18 another toxicity database that contains qualitative results of 617 experiments on 8615 compounds

1941 . The Free Solvation Database (FreeSolv) provides experimental and calculated hydration free

20 energy of 643 small molecules in water ${ }^{41}$. ESOL is a small dataset consisting of water solubility

21 data for 1128 compounds ${ }^{41}$. Lipophilicity is an important feature of drug molecules that affects

22 both membrane permeability and solubility of 4200 compounds ${ }^{41}$. PhysProp consists of 14,176

23 molecules and their corresponding $\log P$ values ${ }^{28}$.

\section{Configuration space}


The Configuration space of GLAM consists of two parts: architecture decisions and training

2 decisions. The architecture decisions decide how to build an architecture of a model. The general

3 architectures of pairs- and single-molecule contain 8 and 4 blocks with their independent design

4 space, respectively, as shown in Figure 1. The training decisions decide how to train the model,

5 including batch size, number of epochs, type of loss, type of optimizer, learning rate, reduce rate

6 of learning rate, reduce patience of learning rate, early-stop patience.

$7 \quad$ Graph learning in architectures

8 Given a molecular graph $G$ with $x_{i}$ denoting node features of node $i$ and $e_{j i}$ denoting edge

9 features from node $j$ to node $i$, the feed-forward block can be described as

$$
h_{i}=f_{n n}\left(x_{i}\right)
$$

11 , where $h_{i}$ denote the hidden node features and $f_{n n}$ denote a feed-forward neural network. And

12 the message-passing block can be described as

$$
h_{i}^{\prime}=f_{u}\left(h_{i}, \sum_{j \in N_{i}} f_{i}\left(h_{j}, e_{j i}\right)\right)
$$

14 where $f_{u}$ denote the update function and $f_{i}$ denote the interaction function. The output property $p$

15 are transformed by the global pooling block and final feed-forward block can be described as

$$
p=f_{n n}\left(f_{\text {pool }}\left(h_{i}^{\prime}\right)\right)
$$

17 where $f_{n n}$ denote a feed-forward neural network and $f_{\text {pool }}$ denote the global pooling layer.

\section{Design spaces of blocks}

19 Each block is created with its own design space, as show in Extended Data Figure 1. The

20 feed-forward block consists of a normalization layer, a dropout layer, a feed-forward layer, and an 
activation layer. The normalization layer, dropout layer, and activation layer can be chosen to be empty in this block. Most parts of the message-passing block are the same as the feed-forward

3 block, but the core is changed to a message-passing layer with a choice of five possible types. The 4 fusion block is designed to extract information on a pair of interacting molecules. Global pool block

5 consists of one layer of graph pool layer with a choice of three types of pooling layers.

\section{Preparing for both molecular interactions and properties prediction}

We prepare two general architectures for both molecular interactions and properties prediction, as shown in the Figure 3. The pair-graph architecture for molecular interactions accepts a pair of molecules as input and outputs their interaction. The single-graph architecture for

10 molecular properties accepts a molecule as input and outputs one or multiple properties of the

11 molecule. Some essential tasks in drug discovery are molecular interactions, such as protein-ligand

12 interactions. All molecules are processed to graphs with basic node attributes as input of the

13 architectures. Small molecules are processed into atom-level molecular graphs, where the edge

14 information is provided by chemical bonds. Proteins are processed into residue-level graphs, where

15 the edge information is provided by contact maps predicted by RaptorX 46.

\section{Multi-GPU parallel}

17 GLAM works in parallel with multiple graphics cards. The most time-consuming parts of a 18 graph learning process are the training, validation, and test. The proposed methodhave lots of 19 independent graph learning processes. Subsequently, we let them work in parallel to utilize the

20 computational source fully. In detail, we build a queue and insert all these processes as jobs into it.

21 If a graphics card is free, a job will be popped up and be assigned to the card until all the jobs are

22 popped up.

\section{Robustness experiments using molecular structure perturbations}

Molecular structure perturbation experiment aims to investigate the robustness of a model. If 
1 the accuracy of a method is not greatly affected by some perturbations, the method is robust enough. A dataset is first divided into training set, validation set and test set. Among them, the

3 training set is used to train a model of the method, the verification set is used to decide when to save the model weights, and the test set is used for robustness experiment. set $P=\left\{p_{i} \mid 1 \leq i \leq N\right\}$ by equation (4). Given a property observer function $o$, we can calculate a property observer set $Q=\left\{q_{i} \mid 1 \leq i \leq N\right\}$ by equation (5).

$$
\begin{aligned}
& P=f(M) \\
& Q=o(M)
\end{aligned}
$$

10 Applying an algorithm $g$ to $\mathrm{M}$, we can obtain a perturbed test set $M^{\prime}=\left\{m_{i}^{\prime} \mid 1 \leq i \leq N^{\prime}\right\}$,

11 where $N^{\prime}$ is the size of molecules that have been applied $g$ successfully. We also predict the 12 property set $P^{\prime}=\left\{p_{i}^{\prime} \mid 1 \leq i \leq N^{\prime}\right\}$ by equation (6). Using the observer function $o$ above, we can

13 calculate a property observer set $Q^{\prime}=\left\{q_{i}^{\prime} \mid 1 \leq i \leq N^{\prime}\right\}$ of $M^{\prime}$ by equation (7). the robustness of a model. Given a loss function $L$, if $L\left(P, P^{\prime}\right)>L\left(Q, Q^{\prime}\right)$, the model is not robust, 18 that is, the perturbation will have an essential impact on performance of the model. If $L\left(P, P^{\prime}\right) \leq$ $19 L\left(Q, Q^{\prime}\right)$, the model is robust and the perturbation will have less impact. We define a perturbation

20 Effect score by equation (8), where the $\Delta(f, g)$ represents the perturbation Effect score of method $21 f$ on the perturbation function $g$.

$$
\Delta(f, g)=L\left(P, P^{\prime}\right)-L\left(Q, Q^{\prime}\right)
$$

\section{Property-slightly-affected structural perturbation}


Property-slightly-affected structural perturbation (PASP) works by iteratively mutating the molecular structure with small perturbations that do not significantly affect the properties of the

3 molecules. In each iteration, PASP mutate the structure of a molecule by modifying/adding a random atom. If the mutated molecule can meet two conditions at the same time in this number of

5 perturbations during the iterations, we got a new perturbed molecular $x_{i}^{\prime}$. The first condition is that

6 the molecular fingerprint similarity $S\left(x_{i}, x_{i}^{\prime}\right) \in\left[\gamma_{\min }, \gamma_{\max }\right)$, where $\left[\gamma_{\min }, \gamma_{\max }\right)$ is a preset

7 similarity area. The second condition is that the loss $L\left(q_{i}, q_{i}^{\prime}\right)<\epsilon_{2}$, where $\epsilon_{1}$ is a preset maximum

8 loss. Finally, we combine all the perturbed molecules into a set $M^{\prime}=\left\{m_{i}^{\prime} \mid 1 \leq i \leq N^{\prime}\right\}$, where $N^{\prime}$

9 is the size of all molecules that be perturbed successfully.

\section{Node-level interpretation}

11 We extract the output of the message-passing block, which is a matrix $X=\left\{x_{i j} \mid 1 \leq i \leq\right.$

$12 N, 1 \leq j \leq M\} . N$ is the number of atoms, and $M$ is the dimension of the outputs. Then we obtain

13 the weight $W=\left\{w_{i} \mid 1 \leq 1 i \leq N\right\}$ of each atom according to $w_{i}=\frac{1}{M} \sum_{j=1}^{M} x_{i j}$, and visualize the

14 molecule with the weights. In some cases, the weight $w_{i}$ may be scaled to $[-1,1]$ or $[0,1]$.

\section{Acknowledgements}

This work was supported by the National Natural Science Foundation of China (21775060).

19 We acknowledge that the work in this paper was performed at the high-performance computing

20 resources from Supercomputing Center of Lanzhou University. We thank the help from Prof. Jinbo

$21 \mathrm{Xu}$, the author of RaptorX. We thank the help from Mingjian Jiang, the author of DGraphDTA.

\section{Author Contributions}



and conducted the experiments. C.H., S.L. , Y.T. , D.J., J.Y., Q.B., and H.L. evaluated the

3 experiments and contributed ideas. S.Z., C.H. and X.Y. managed and supervised the project. All

4 authors co-wrote the manuscript.

5 Competing Interests Statement

The authors declare no competing interests.

\section{Data availability}

All data used in this paper are publicly available and can be accessed at http://drugdesign.unistra.fr/LIT-PCBA/ for LIT-PCBA dataset. https://github.com/lifanchen-

$10 \mathrm{simm} /$ transformerCPI for BindingDB dataset, https://github.com/kexinhuang12345/CASTER for

11 the DrugBank dataset, and http://moleculenet.ai/datasets-1 for all molecules datasets.

\section{Code availability}

All code of GLAM is available at https://github.com/yvquanli/GLAM.

\section{References}

17 1. Schneider, G. Automating drug discovery. Nature Reviews Drug Discovery 17, 97-113

18 (2018).

19 2. Schneider, P. et al. Rethinking drug design in the artificial intelligence era. Nature Reviews

Drug Discovery 19, 353-364 (2020).

3. Inglese, J. \& Auld, D. S. High Throughput Screening (HTS) Techniques: Applications in Chemical Biology. in Wiley Encyclopedia of Chemical Biology (2008). doi:10.1002/9780470048672.wecb223.

4. Sliwoski, G., Kothiwale, S., Meiler, J. \& Lowe, E. W. Computational methods in drug discovery. Pharmacological Reviews vol. 66 334-395 (2014).

5. Fleming, N. How artificial intelligence is changing drug discovery. Nature 557, S55-S57 (2018).

6. Zheng, S., Li, Y., Chen, S., Xu, J. \& Yang, Y. Predicting drug-protein interaction using quasi-visual question answering system. Nature Machine Intelligence 2, 134-140 (2020). 
7. Shen, W. X. et al. Out-of-the-box deep learning prediction of pharmaceutical properties by broadly learned knowledge-based molecular representations. Nature Machine Intelligence (2021) doi:10.1038/s42256-021-00301-6.

8. Kotsias, P.-C. et al. Direct steering of de novo molecular generation with descriptor conditional recurrent neural networks. Nature Machine Intelligence 2, 254-265 (2020).

9. Méndez-Lucio, O., Baillif, B., Clevert, D. A., Rouquié, D. \& Wichard, J. De novo generation of hit-like molecules from gene expression signatures using artificial intelligence. Nature Communications 11, 1-10 (2020).

10. Chen, H., Engkvist, O., Wang, Y., Olivecrona, M. \& Blaschke, T. The rise of deep learning in drug discovery. Drug Discovery Today 23, 1241-1250 (2018).

11. Jiang, S. \& Balaprakash, P. Graph Neural Network Architecture Search for Molecular Property Prediction. arXiv (2020) doi:10.1109/bigdata50022.2020.9378060.

12. Cai, S. et al. Rethinking Graph Neural Architecture Search from Message-passing. (2021).

13. Zhang, Z., Wang, X. \& Zhu, W. Automated Machine Learning on Graphs: A Survey. (2021).

14. He, X., Zhao, K. \& Chu, X. AutoML: A survey of the state-of-the-art. Knowledge-Based Systems 212, (2021).

15. Ekins, S. et al. Exploiting machine learning for end-to-end drug discovery and development. Nature Materials 18, 435-441 (2019).

16. Sculley, D. et al. Hidden technical debt in machine learning systems. in Advances in Neural Information Processing Systems vols. 2015-Janua 2503-2511 (2015).

17. Jiang, M. et al. Drug-target affinity prediction using graph neural network and contact maps. RSC Advances 10, 20701-20712 (2020).

18. Kipf, T. N. \& Welling, M. Semi-supervised classification with graph convolutional networks. in International Conference on Learning Representations (2017).

19. Veličković, P. et al. Graph attention networks. International Conference on Learning Representations 1-12 (2018).

20. Gilmer, J., Schoenholz, S. S., Riley, P. F., Vinyals, O. \& Dahl, G. E. Neural message passing for quantum chemistry. in International Conference on Machine Learning vol. 3 2053-2070 (2017).

21. Xiong, Z. et al. Pushing the boundaries of molecular representation for drug discovery with graph attention mechanism. Journal of Medicinal Chemistry acs.jmedchem.9b00959 (2019) doi:10.1021/acs.jmedchem.9b00959.

22. Xu, K., Jegelka, S., Hu, W. \& Leskovec, J. How powerful are graph neural networks? in 7th International Conference on Learning Representations, ICLR 2019 (2019).

23. Li, H., Leung, K. S., Wong, M. H. \& Ballester, P. J. Improving autodock vina using random forest: The growing accuracy of binding affinity prediction by the effective exploitation of larger data sets. Molecular Informatics 34, 115-126 (2015).

24. Chen, L. et al. TransformerCPI: Improving compound-protein interaction prediction by sequence-based deep learning with self-attention mechanism and label reversal experiments. Bioinformatics (2020) doi:10.1093/bioinformatics/btaa524.

25. Huang, K., Xiao, C., Hoang, T., Glass, L. \& Sun, J. CASTER: Predicting Drug Interactions with Chemical Substructure Representation. Proceedings of the AAAI Conference on Artificial Intelligence 34, 702-709 (2020).

26. Yang, Y.-Y., Rashtchian, C., Zhang, H., Salakhutdinov, R. \& Chaudhuri, K. A Closer Look at Accuracy vs. Robustness. 1-14 (2020).

27. Qayyum, A., Qadir, J., Bilal, M. \& Al-Fuqaha, A. Secure and Robust Machine Learning for Healthcare: A Survey. IEEE Reviews in Biomedical Engineering vol. 14 156-180 (2021). 
28. Tetko, I. v., Tanchuk, V. Y. \& Villa, A. E. P. Prediction of n-Octanol/Water Partition Coefficients from PHYSPROP Database Using Artificial Neural Networks and E-State Indices. Journal of Chemical Information and Computer Sciences 41, 1407-1421 (2001).

29. Landrum, G. RDKit : A software suite for cheminformatics , computational chemistry, and predictive modeling. https://www.rdkit.org/ (2011).

30. Zeng, Y., Chen, X., Luo, Y., Li, X. \& Peng, D. Deep drug-target binding affinity prediction with multiple attention blocks. Briefings in Bioinformatics 00, 1-10 (2021).

31. Withnall, M., Lindelöf, E., Engkvist, O. \& Chen, H. Building attention and edge message passing neural networks for bioactivity and physical-chemical property prediction. Journal of Cheminformatics 12, 1-18 (2020).

32. Xiong, J., Xiong, Z., Chen, K., Jiang, H. \& Zheng, M. Graph neural networks for automated de novo drug design. Drug Discovery Today 26, 1382-1393 (2021).

33. Dai, H. et al. Retrosynthesis Prediction with Conditional Graph Logic Network. in NeurIPS $1-11(2020)$.

34. Wang, X. et al. RetroPrime: A Diverse, plausible and Transformer-based method for SingleStep retrosynthesis predictions. Chemical Engineering Journal 420, 129845 (2021).

35. Kuznetsov, M. \& Polykovskiy, D. MolGrow: A Graph Normalizing Flow for Hierarchical Molecular Generation. AAAI 2021 (2021).

36. Luo, Y., Yan, K. \& Ji, S. GraphDF: A Discrete Flow Model for Molecular Graph Generation. ICML 2021 (2021).

37. Liu, M., Yan, K., Oztekin, B. \& Ji, S. GraphEBM: Molecular Graph Generation with EnergyBased Models. ILCR workshop on energy based models 1-16 (2021).

38. Tran-Nguyen, V. K., Jacquemard, C. \& Rognan, D. LIT-PCBA: An unbiased data set for machine learning and virtual screening. Journal of Chemical Information and Modeling 60, 4263-4273 (2020).

39. Gilson, M. K. et al. BindingDB in 2015: A public database for medicinal chemistry, computational chemistry and systems pharmacology. Nucleic Acids Research 44, D1045D1053 (2016).

40. Wishart, D. S. et al. DrugBank: A knowledgebase for drugs, drug actions and drug targets. Nucleic Acids Research 36, (2008).

41. Wu, Z. et al. MoleculeNet: A benchmark for molecular machine learning. Chemical Science 9, 513-530 (2018).

42. Template-based protein structure modeling using the RaptorX web server. (2012) doi:10.1016/j.physbeh.2017.03.040.

43. Halgren, T. A. et al. Glide: A New Approach for Rapid, Accurate Docking and Scoring. 2. Enrichment Factors in Database Screening. Journal of Medicinal Chemistry 47, 1750-1759 (2004).

44. Fey, Matthias and Lenssen, J. E. Fast Graph Representation Learning with PyTorch Geometric. in International Conference on Learning Representations (2019). 
Table 1. Performance comparison on datasets of molecular interactions

\begin{tabular}{ccccccc}
\hline \multirow{2}{*}{$\begin{array}{c}\text { Dataset } \\
\text { Metrics }\end{array}$} & ALDH1 & $\begin{array}{c}\text { ESR1_ant } \\
\text { AUC(higher is better) }\end{array}$ & KAT2A & MAPK1 & BindingDB & $\begin{array}{c}\text { Params } \\
\text { adjust on }\end{array}$ \\
\hline Glide (SP) & $0.607(-)$ & $0.590(-)$ & $0.474(-)$ & $0.592(-)$ & - & - \\
\cline { 2 - 7 } Glide (XP) & $0.582(-)$ & $0.540(-)$ & $0.441(-)$ & $0.579(-)$ & - & - \\
\cline { 2 - 7 } RFScore V3 & $0.556(-)$ & $0.562(-)$ & $0.511(-)$ & $0.640(-)$ & - & - \\
\cline { 2 - 7 } & $0.679(0.007)$ & $0.603(0.022)$ & $0.633(0.017)$ & $0.654(0.020)$ & $0.914(0.027)$ & ALDH1 \\
DGraphDTA & $0.673(0.013)$ & $0.610(0.011)$ & $0.599(0.032)$ & $0.665(0.031)$ & $0.921(0.023)$ & BindingDB \\
\cline { 2 - 7 } & $0.694(0.008)$ & $0.590(0.010)$ & $0.633(0.022)$ & $0.683(0.008)$ & $0.926(0.017)$ & ALDH1 \\
TransformerCPI & $0.665(0.008)$ & $0.616(0.032)$ & $0.650(0.042)$ & $0.662(0.012)$ & $0.937(0.016)$ & BindingDB \\
\cline { 2 - 7 } GLAM & $\mathbf{0 . 7 6 1 ( 0 . 0 0 6 )}$ & $\mathbf{0 . 6 6 6 ( 0 . 0 1 2 )}$ & $\mathbf{0 . 7 0 9 ( 0 . 0 3 3 )}$ & $\mathbf{0 . 7 3 0}(0.016)$ & $\mathbf{0 . 9 5 4 ( 0 . 0 0 8 )}$ & None \\
\hline
\end{tabular}

2 Note: (a) All datasets are split randomly. (b) Glide ${ }^{43}$ docking scores are obtained on Schrödinger version 32015 with the precision of SP and XP. (c) DGraphDTA ${ }^{17}$, TransformerCPI ${ }^{24}$ are implemented from their 4 open-source code. Their hyper-parameters have been adjusted to obtain the best performance. (d) All deep 5 learning methods are run with three different split seeds, and then we take the average score and the standard 6 deviation (in parentheses). (e) the highlight text with bold-black style means the best.

7 Table 2. Performance comparison on datasets of molecular properties

\begin{tabular}{|c|c|c|c|c|c|c|c|c|c|}
\hline \multirow{3}{*}{$\begin{array}{c}\text { Task } \\
\text { Dataset } \\
\text { Metrics }\end{array}$} & \multicolumn{3}{|c|}{ Physical chemistry } & \multirow{2}{*}{$\frac{\text { Bioactivity }}{\text { BACE }}$} & \multicolumn{2}{|c|}{ Pharmacokinetics } & \multicolumn{2}{|c|}{ Toxicity } & \multirow{3}{*}{$\begin{array}{c}\text { Params } \\
\text { adjust on }\end{array}$} \\
\hline & ESOL & Lipophilicity & FreeSolv & & BBBP & SIDER & Tox21 & ToxCast & \\
\hline & \multicolumn{3}{|c|}{ RMSE (lower is better) } & \multicolumn{5}{|c|}{ AUC (higher is better) } & \\
\hline \multirow{2}{*}{ GCN } & $1.017(0.064)$ & $0.807(0.044)$ & $2.307(0.147)$ & $0.772(0.050)$ & $0.830(0.057)$ & $0.619(0.028)$ & $0.762(0.026)$ & $0.678(0.007)$ & ESOL \\
\hline & $1.056(0.096)$ & $0.799(0.062)$ & $2.858(0.524)$ & $0.797(0.018)$ & $0.792(0.083)$ & $0.612(0.030)$ & $0.752(0.030)$ & $0.663(0.024)$ & BACE \\
\hline \multirow{2}{*}{ GAT } & $1.079(0.080)$ & $0.925(0.031)$ & $2.491(0.465)$ & $0.716(0.033)$ & $0.815(0.067)$ & $0.606(0.053)$ & $0.769(0.033)$ & $0.675(0.013)$ & ESOL \\
\hline & $1.188(0.058)$ & $0.834(0.037)$ & $2.343(0.272)$ & $0.759(0.019)$ & $0.842(0.042)$ & $0.613(0.023)$ & $0.713(0.022)$ & $0.687(0.017)$ & BACE \\
\hline \multirow{2}{*}{ GIN } & $0.704(0.078)$ & $0.948(0.058)$ & $2.662(0.289)$ & $0.812(0.032)$ & $0.858(0.035)$ & $0.595(0.031)$ & $0.793(0.026)$ & $0.671(0.019)$ & ESOL \\
\hline & $0.742(0.058)$ & $0.864(0.044)$ & $2.098(0.272)$ & $0.831(0.040)$ & $0.875(0.019)$ & $0.605(0.040)$ & $0.732(0.019)$ & $0.672(0.016)$ & BACE \\
\hline \multirow{2}{*}{ MPNN } & $0.755(0.077)$ & $0.769(0.031)$ & $1.897_{(0.092)}$ & $0.820(0.047)$ & $0.831(0.036)$ & $0.626(0.038)$ & $0.752(0.011)$ & $0.697(0.023)$ & ESOL \\
\hline & $0.884(0.061)$ & $0.825(0.047)$ & $2.038(0.421)$ & $0.816(0.042)$ & $0.816(0.057)$ & $0.641(0.014)$ & $0.803(0.031)$ & $0.686(0.017)$ & BACE \\
\hline \multirow{2}{*}{ AttentiveFP } & $0.726(0.032)$ & $0.724(0.030)$ & $1.775(0.392)$ & $0.815(0.072)$ & $0.856(0.023)$ & $0.654(0.027)$ & $0.763(0.022)$ & $0.726(0.020)$ & ESOL \\
\hline & $0.738(0.059)$ & $0.783(0.036)$ & $1.371(0.446)$ & $0.850(0.017)$ & $0.872(0.024)$ & $0.621(0.033)$ & $0.740(0.039)$ & $0.680(0.024)$ & BACE \\
\hline MolMapNet & $0.752(0.040)$ & $0.731(0.012)$ & $1.398(0.312)$ & $0.868(0.094)$ & $0.911(0.013)$ & $0.634(0.015)$ & $0.813(0.021)$ & $0.703(0.025)$ & None \\
\hline GLAM & $\mathbf{0 . 5 9 2}(0.036)$ & $\mathbf{0 . 5 9 6}(0.025)$ & $\mathbf{1 . 3 1 9}_{(0.346)}$ & $\mathbf{0 . 8 8 8}_{(0.033)}$ & $\mathbf{0 . 9 3 2}_{(0.015)}$ & $\mathbf{0 . 6 5 9}(0.017)$ & $\mathbf{0 . 8 4 1}(0.010)$ & $\mathbf{0 . 7 4 4}(0.008)$ & None \\
\hline
\end{tabular}

8 Note: (a) All datasets are split by scaffold. (b) $\mathrm{GCN}^{18}, \mathrm{GAT}^{19}, \mathrm{GIN}^{22}, \mathrm{MPNN}^{20}$ are implemented with

9 PyTorch Geometric ${ }^{44}$. AttentiveFP ${ }^{21}$ is implemented from its open-source code. Their hyper-parameters

10 have been adjusted to obtain the best performance. (c) All methods are run with three different split seeds, 
1 and then we take the average score and the standard deviation (in parentheses). (d) the highlight text with 2 bold-black style means the best.

3
Table 3. Effect score of molecular structure perturbation test

\begin{tabular}{cccc}
\hline Perturbation Level & Level 1 & Level 2 & Level 3 \\
Fingerprint Similarity & $\mathbf{0 . 8 - 1 . 0}$ & $\mathbf{0 . 5 - 0 . 8}$ & $\mathbf{0 . 3 - 0 . 5}$ \\
Metrics & \multicolumn{2}{c}{ Effect score (lower is better) } \\
\hline GCN & $0.728( \pm 0.019)$ & $0.929( \pm 0.015)$ & $1.092( \pm 0.009)$ \\
GAT & $0.750( \pm 0.022)$ & $0.985( \pm 0.118)$ & $1.149( \pm 0.124)$ \\
GIN & $0.695( \pm 0.064)$ & $0.953( \pm 0.100)$ & $1.087( \pm 0.074)$ \\
MPNN & $0.598( \pm 0.018)$ & $0.820( \pm 0.069)$ & $0.998( \pm 0.083)$ \\
GLAM & $\mathbf{0 . 3 6 0}( \pm \mathbf{0 . 0 1 4})$ & $\mathbf{0 . 4 6 9}( \pm \mathbf{0 . 0 1 9})$ & $\mathbf{0 . 6 3 2}( \pm \mathbf{0 . 0 1 3})$
\end{tabular}

5 Note: (a)All baselines above are all implemented with PyTorch Geometric ${ }^{44}$, and their hyper-parameters have been manually

6 adjusted for several rounds. (b) The losses between $\mathrm{P}$ and $\mathrm{P}^{\prime}$ of level 1,2,3 perturbated test sets are 0.0624, 0.0593, 0.0578,

7 respectively.

8 


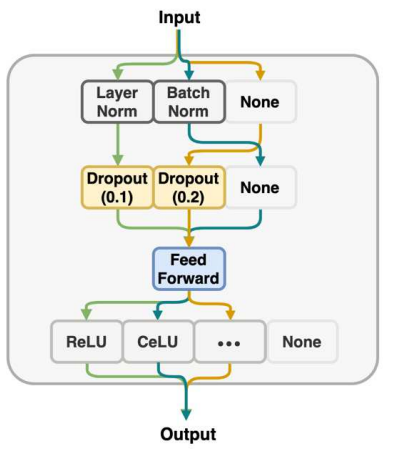

Feed-forward Block

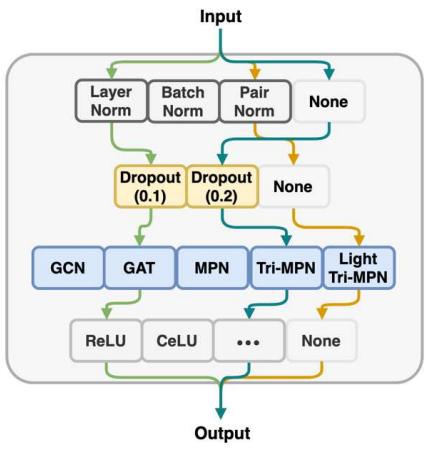

Message Passing Block
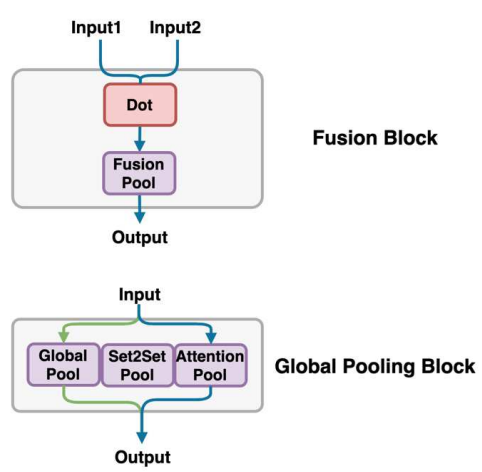

2 Extended Data Figure 1. design space for blocks of the architecutures.

a

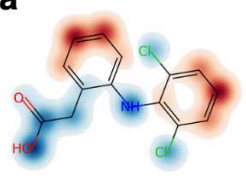

pred: 0.69 true: $\mathbf{0 . 6 5}$

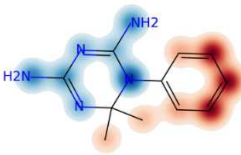

pred: $\mathbf{0 . 4 2}$ true: $\mathbf{0 . 3 9}$

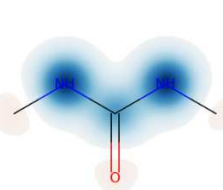

pred: 0.43 true: 0.49

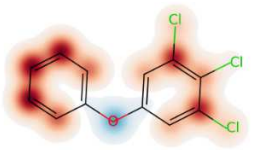

pred: $\mathbf{5 . 5 4}$ true: $\mathbf{5 . 7 0}$
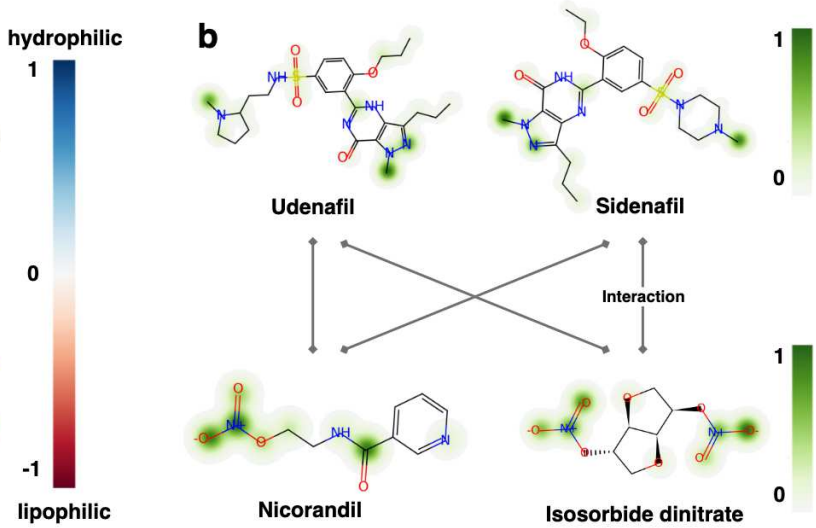

3

4 Extended Data Figure 2. Node-level interpretation. a, case studies of solubility prediction. b case

$5 \quad$ studies of drug-drug interactions.

Extended Data Table 1. Performance comparison on drug-drug interaction datasets

\begin{tabular}{ccc}
\hline Dataset & \multicolumn{2}{c}{ DrugBank } \\
\hline Metrics & ROC-AUC & PR-AUC \\
LR & $0.774( \pm 0.003)$ & $0.745( \pm 0.005)$ \\
Nat.Prot & $0.786( \pm 0.003)$ & $0.753( \pm 0.003)$ \\
Mol2Vec & $0.849( \pm 0.004)$ & $0.828( \pm 0.006)$ \\
MOLVAE & $0.852( \pm 0.006)$ & $0.828( \pm 0.009)$ \\
DeepDDI & $0.844( \pm 0.003)$ & $0.828( \pm 0.002)$ \\
CASTER & $0.861( \pm 0.005)$ & $0.829( \pm 0.003)$ \\
GLAM & $\mathbf{0 . 9 1 1}( \pm \mathbf{0 . 0 1 7})$ & $\mathbf{0 . 8 9 9}( \pm \mathbf{0 . 0 1 9})$
\end{tabular}

7 Note (a) In the drug-drug interaction task, scores of all baselines are taken from CASTER ${ }^{25}$. (b) the highlight

8 score with bold-black style means this method got the highest score. (c) all scores of GLAM are run under

9 the same default configuration setting. 